\title{
Ocorrência de Glyphonycteris sylvestris Thomas (Chiroptera, Phyllostomidae) no Estado do Rio de Janeiro, sudeste do Brasil
}

\author{
Daniela Dias ${ }^{1}$, Shirley Seixas Pereira da Silva ${ }^{1,2} \&$ Adriano Lúcio Peracchi ${ }^{1}$
}

\author{
${ }^{1}$ Laboratório de Mastozoologia, Instituto de Biologia, Universidade Federal Rural do Rio de Janeiro. Antiga Rodovia Rio São \\ Paulo, Km 47, 23890-000 Seropédica, Rio de Janeiro, Brasil. E-mail: ddtdani@ig.com.br \\ ${ }^{2}$ Fundação Instituto Estadual de Florestas. Avenida 13 de Maio, 26ª andar, Centro, 20031-000 Rio de Janeiro, Rio de \\ Janeiro, Brasil.
}

\begin{abstract}
Occurrence of Glyphonycteris sylvestris Thomas (Chiroptera, Phyllostomidae) in Rio de Janeiro State, Southeastern Brazil. The first occurrence of phyllostomid bat Glyphonycteris sylvestris Thomas, 1896 in Rio de Janeiro State, Southeastern Brazil is reported. An adult female was caught with mist net in the Parque Estadual da Pedra Branca, an Atlantic Forest area in West Zone of Rio de Janeiro city, in July $9^{\text {th }}$, 1996. Morphological aspects of this species are discussed. Measurements obtained for the specimen are also provided.
\end{abstract}

KEY WORDS. Atlantic Forest, Chiroptera, Glyphonycteris sylvestris, Phyllostomidae, Southeastern Brazil.

Em 09 de julho de 1996, durante um levantamento de espécies de morcegos conduzido no Parque Estadual da Pedra Branca (PEPB) $\left(23^{\circ} 52^{\prime}\right.$ e $23^{\circ} 04^{\prime} \mathrm{S}$; $43^{\circ} 23^{\prime}$ e $\left.43^{\circ} 32^{\prime} \mathrm{W}\right)$, Zona Oeste do município do Rio de Janeiro, Estado do Rio de Janeiro (Dias et al. 2002), foi colecionada uma fêmea adulta de Glyphonycteris sylvestris Thomas, 1896. O exemplar foi capturado em rede de espera ("mist-net") armada ao nível do solo em uma trilha na mata, e encontra-se incorporado à Coleção Adriano Lúcio Peracchi (ALP), atualmente depositada no Instituto de Biologia da Universidade Federal Rural do Rio de Janeiro (UFRRJ), na forma de pele cheia, sob a numeração ALP 5856.

Segundo SANBorn (1949), os morcegos dos gêneros Micronycteris Gray, 1866, Glyphonycteris Thomas, 1896 e Xenoctenes Miller, 1907 foram originalmente reunidos no gênero Schizostoma Gervais, 1856. Entretanto, esse nome era pré-ocupado por Schizostoma Bronn, 1835, um gênero de Mollusca e esse autor considerou que as relações entre estes gêneros poderiam ser mais claramente indicadas se fossem considerados como subgêneros de Micronycteris Gray, 1866, então o nome genérico mais antigo disponível. Além disso, SANBorn (1949) descreveu os subgêneros Lampronycteris, Neonycteris e Trinycteris e forneceu uma chave para identificação dos subgêneros reconhecidos.

Simmons \& Voss (1998), com base em análises filogenéticas, sugeriram que Micronycteris "sensu strictu", bem como os demais subgêneros sejam restaurados como gêneros válidos. Assim, atualmente cinco gêneros e 15 espécies são reconhecidos, a saber: Glyphonycteris Thomas, 1896 (G. behni (Peters, 1865); G. daviesi
(Hill, 1964) e G. sylvestris Thomas, 1896); Lampronycteris Sanborn, 1949 (L. brachyotis (Dobson, 1878)); Micronycteris Gray, 1866 (M. brosseti Simmons \& Voss, 1998; M. homezi Pirlot, 1967; M. hirsuta (Peters, 1869); M. matses Simmons, Voss \& Fleck, 2002; M. megalotis (Gray, 1842); M. microtis Miller, 1907; M. minuta (Gervais, 1856); M. sanborni Simmons, 1996 e M. schmidtorum Sanborn, 1935); Neonycteris Sanborn, 1949 (N. pusilla (Sanborn, 1949)) e Trinycteris Sanborn, 1949 (T. nicefori (Sanborn, 1949)).

Glyphonycteris distingue-se dos demais gêneros pela seguinte combinação de caracteres: orelhas não conectadas por banda; quarto metacarpo mais curto, o quinto é o mais longo; segunda falange do terceiro dedo mais longa; segunda falange do quarto dedo mais longa que a primeira; crânio com caixa craniana alta e processos coronóides baixos; borda superior do ramo ascendente quase horizontal; incisivos superiores internos em forma de cinzel e externos reduzidos; caninos superiores relativamente curtos; primeiro pré-molar superior com cúspide anterior; segundo pré-molar superior com ponta ligeiramente recurvada e incisivos inferiores trilobulados; G. sylvestris distingue-se de $G$. behni e $G$. daviesi principalmente pelo menor comprimento de antebraço, pêlos dorsais tricoloridos (banda basal castanho escura, seguida por banda central branca e extremidades da mesma cor da banda basal) e pêlos ventrais de coloração marrom escura na base e acinzentada na extremidade (cf. Sanborn 1949, Genoways \& Williams 1986, Koopman 1994, SimMons \& Voss 1998).

Revista Brasileira de Zoologia 20 (2): 365-366, junho 2003 
Todos os caracteres morfológicos (externos, cranianos e dentários) acima citados como diagnósticos para Glyphonycteris e G. sylvestris foram observadas no exemplar em questão, o que permitiu sua identificação segura. Medidas (em milímetros) obtidas da fêmea colecionada são: comprimento de antebraço 41,1; terceiro metacarpo 37,0; quarto metacarpo 35,9; quinto metacarpo 38,7; comprimento total do crânio 20,2; comprimento côndilo-basal 18,5; comprimento basal 16,3; comprimento palatal 9,4; comprimento da série de dentes superiores 8,1; comprimento da mandíbula 13,1; comprimento da série de dentes inferiores 8,5; largura externa entre os molares 6,5; largura entre os cingula caninos 3,6; largura pós-orbital 4,7; largura zigomática 10,0; largura mastóidea 9,0; largura da caixa craniana 8,4 .

Segundo Koopman (1993), G. sylvestris distribui-se pelo Peru e sudeste do Brasil até Nayarit e Veracruz (México) e Trinidad. No território brasileiro, essa espécie tem sido registrada na Amazônia (Bernard 2002), no Estado do Paraná, Sul do Brasil (Sekiama et al. 2001) e no Estado de São Paulo (Trajano 1982, Pedro et al. 2001). Marinho-Filho (1996) mencionou a ocorrência dessa espécie no Rio de Janeiro, mas não forneceu dados sobre localidade exata nem número de registro em coleção de qualquer exemplar. O exemplar do PEPB constitui, portanto, o primeiro registro comprovado para o Estado do Rio de Janeiro.

Além de G. sylvestris, outras espécies de morcegos amostradas no Parque Estadual da Pedra Branca (DiAs et al. 2002) foram: Chrotopterus auritus (Peters, 1856); Micronycteris megalotis (Gray, 1842); Micronycteris minuta (Gervais, 1856); Mimmon bennettii (Gray, 1838); Phyllostomus hastatus (Pallas, 1767); Tonatia bidens (Von Spix, 1823); Lonchophylla bokermanni Sazima, Vizotto \& Taddei, 1978; Lonchophylla mordax Thomas, 1903; Anoura caudifera (E. Geoffroy, 1818); Glossophaga soricina (Pallas, 1766); Carollia perspicillata (Linnaeus, 1758); Artibeus fimbriatus Gray, 1838; Artibeus lituratus (Olfers, 1818); Artibeus obscurus Schinz, 1821; Chiroderma doriae Thomas, 1891; Platyrrhinus lineatus (E. Geoffroy, 1810); Platyrrhinus recifinus (Thomas, 1901); Sturnira lilium (E. Geoffroy, 1810); Vampyressa pusilla (Wagner, 1843); Desmodus rotundus (E. Geoffroy, 1810); Diphylla ecaudata Von Spix, 1823; Eptesicus brasiliensis (Desmarest, 1819); Myotis nigricans (Schinz, 1821) e Molossus molossus (Pallas, 1766).

\section{AGRADECIMENTOS}

Somos gratos aos biólogos Vítor de Souza Ferreira, Alexandre Pinhão da Cruz, Gustavo Amaral Borges, Fabrício Olímpio de Lima e André Mariz da Silva, pelo auxílio nos trabalhos de campo e de laboratório; à Administração do Parque Estadual da Pedra Branca (Fundação Instituto Estadual de Florestas, Rio de Janeiro), pela autorização e oportunidade de realizar trabalhos de campo na área do Parque e, ainda, aos servidores do Instituto de Biologia da Universidade Federal Rural do Rio de Janeiro, pelo auxílio nos momentos necessários.

\section{REFERÊNCIAS BIBLIOGRÁFICAS}

BERNARD, E. 2002. Diet, activity and reproduction of bat species (Mammalia, Chiroptera) in Central Amazonia, Brazil. Revista Brasileira de Zoologia, Curitiba, 19 (1): 173-188.

Dias, D., A.L. Peracchi \& S.S.P. DA Silva. 2002. Quirópteros do Parque Estadual da Pedra Branca, Rio de Janeiro, RJ (Mammalia: Chiroptera). Revista Brasileira de Zoologia, Curitiba, 19 (Supl. 2): 113-140.

Genoways, H.H. \& S.L. Williams. 1986. Results of the Alcoa Foundation - Suriname Expeditions. XI. Bats of the genus Micronycteris (Mammalia: Chiroptera) in Suriname. Annals of the Carnegie Museum of Natural History, Pittsburgh, 55: 303-324.

Koopman, K.F. 1993. Order Chiroptera, p.137-241. In: D.E. WILSON \& D.M. REEDER (Eds). Mammal species of the world: a taxonomic and geografic reference. Washington, D.C., Smithsonian Institution Press, $2^{\text {nd }}$ ed., XVIII+1206p. . 1994. Chiroptera: systematics. Handbuch der Zoologie [Handbook of Zoology], VIII (Mammalia) (60). Berlin and New York, Walter de Gruyter, VII+217p.

Marinho-Filho, J. 1996. Distribution of bat diversity in the southern and southeastern Brazilian Atlantic Forest. Chiroptera Neotropical, Brasília, 2 (2): 51-54.

Pedro, W.A.; F.C. Passos \& B.K. Lim. 2001. Morcegos (Chiroptera; Mammalia) da Estação Ecológica dos Caetetus, Estado de São Paulo. Chiroptera Neotropical, Brasília, 7 (1-2): 136140.

SAnborn, C.C. 1949. Bats of the genus Micronycteris and its subgenera. Fieldiana Zoology, Chicago, 31: 215-233.

Sekiama, M.L.; N.R. dos Reis; A.L. Peracchi \& V.J. Rocha. 2001. Morcegos do Parque Nacional do Iguaçu, Paraná (Chiroptera, Mammalia). Revista Brasileira de Zoologia, Curitiba, 18 (3): 749-754.

Simmons, N.B. \& R.S. Voss. 1998. The mammals of Paracou, French Guiana: a neotropical lowland rainforest fauna. Part I. Bats. Bulletin of the American Museum of Natural History, New York, 273: 1-219.

Trajano, E. 1982. New records of bats from Southeastern Brazil. Journal of Mammalogy, Lawrence, 63 (3): 529.

Recebido em 05.XII.2002; aceito em 27.V.2003. 